\title{
Incentive learning and the motivational control of instrumental performance by thirst
}

\author{
MATIAS LOPEZ \\ Universidad de Oviedo, Oviedo, Spain \\ and \\ BERNARD BALLEINE and ANTHONY DICKINSON \\ University of Cambridge, Cambridge, England
}

\begin{abstract}
The role of incentive learning in instrumental performance following a shift in the degree of water deprivation was analyzed in three experiments. In Experiments 1A and 1B, rats trained to perform an instrumental action reinforced with either sucrose or maltodextrin solutions when in a high-deprivation state were subsequently shifted to a low-deprivation state and tested in extinction. This within-state shift in water deprivation reduced instrumental performance only when the animals had been exposed to the reinforcer in the low-deprivation state prior to instrumental training. In Experiment 2, a concurrent training procedure was used to assess whether the change in the value of the reinforcer brought about by preexposure was mediated by the contingency between the instrumental action and the reinforcer. Preexposure to the reinforcer under the low-deprivation state produced a selective reduction of the performance of the action upon which it was contingent during training when testing was conducted in extinction following a shift from the high- to the low-deprivation state. These experiments provide evidence that animals have to learn about the incentive value of a reinforcer in a particular motivational state through exposure to the reinforcer in that state.
\end{abstract}

Expectancy theories of instrumental conditioning assume that the performance of goal-directed actions is determined not only by the agent's knowledge about actionreinforcer relationships but also by the current incentive value that agents assign to the reinforcer (see Dickinson, 1989). In most cases, however, such theories are usually silent about the processes by which this assignment occurs. In the case of the control of performance by a primary motivational state, Tolman (1949a, 1949b) argued that agents have to learn about the incentive value of a reinforcer through direct, consummatory contact with it in that state, a process that has been referred to as incentive learning by Dickinson and Dawson $(1988,1989)$. Evidence for this process of incentive learning comes from recent studies of both between- and within-state shifts in primary motivation.

To analyze the effect of a shift from hunger to thirst, Dickinson and Dawson $(1988,1989)$ trained hungry rats to press a lever for either a sucrose solution or food pellets (another action, chain pulling, was also trained concurrently with the other reinforcer). The motivational state of the animals was then shifted from hunger to thirst be-

This research was supported by a grant from the DGICYT (MEC, Spain) to Matias Lopez and an SERC project grant to Anthony Dickinson. Correspondence concerning this article should be addressed either to Anthony Dickinson at the Department of Experimental Psychology, University of Cambridge, Downing Street, Cambridge, CB2 3EB, England, or to Matias Lopez, Departamento de Psicologia, Universidad de Oviedo, C/Aniceto Sela s/n, 33005-Oviedo, Spain. fore the propensity to press the lever was assessed in extinction. Dickinson and Dawson argued that, first, if the animals learned about the action-reinforcer contingency during training and, second, if the incentive value of a reinforcer is determined by the agent's current motivational state, then expectancy theory should predict relatively more leverpressing in the extinction test under thirst when the animals had been trained to leverpress with the sucrose solution than when they had been trained with the food pellets. This is because the sucrose solution, being relevant to the state of thirst, should be assigned a higher incentive value than should the food pellets. In agreement with this prediction, Dickinson and Dawson found that their rats pressed the lever more when tested under thirst if they had been trained to leverpress with the sucrose solution rather than the food pellets. This difference was only observed, however, if the animals had previously had the opportunity to consume the two incentives while they were thirsty. In the absence of such experience, performance during the extinction test was unaffected by the type of training reinforcer. Thus, these studies suggest that the motivational control of instrumental performance following a hunger-to-thirst transition depends on a process of incentive learning by which the animal assigns an incentive value to a reinforcer; that value is conditional upon the animal's current motivational state, and it is assigned on the basis of the animal's direct consummatory experience with the reinforcer in that state.

Recently, Balleine (in press) has demonstrated a role for incentive learning in the case of shifts within, as op- 
posed to between, motivational states. He analyzed the influence of a posttraining shift in the degree of food deprivation on the performance of an instrumental action in extinction. In order to assess whether instrumental performance following a posttraining shift from a high to a low level of primary motivation is mediated by incentive learning, Balleine trained hungry rats to press a lever for standard Noyes food pellets. Prior to instrumental training, half of the animals had received presentations of the pellets in the absence of any food deprivation. Immediately after the final instrumental training session, half of the preexposed and half of the nonpreexposed animals were given free access to food in their home cages while the remainder were maintained on a 22.5 -h food deprivation schedule, following which leverpress performance was assessed in an extinction test. Balleine found that a posttraining decrease in motivation level had no effect on subsequent extinction performance in the absence of preexposure to the reinforcer in the low-deprivation state. If, however, animals were allowed to contact the instrumental reinforcer in the test deprivation state prior to training, their performance was altered appropriately when they were shifted to that state following training. More specifically, following a posttraining shift from a high to a low level of food deprivation, the animals that had been given no preexposure to the reinforcer under the low-deprivation state pressed at a rate equivalent to that of animals both trained and tested hungry. By contrast, animals that had been preexposed to the reinforcer under the low level of deprivation, when tested in this low state, pressed at a lower rate than did the remaining groups. Balleine also reported the same pattern of results when the training reinforcer was a polysaccharide solution, maltodextrin, rather than food pellets.

Interpreted in terms of incentive learning, exposure to a reinforcer when animals are hungry during training results in the assignment of a relatively high incentive value. In the absence of any prior experience with the reinforcer in the nondeprived state, animals have no basis on which to change the assigned incentive value when they are shifted to the low-deprivation state for the extinction test. Consequently, performance during this test, although the animals are nondeprived, is controlled by the high value assigned during training. Preexposure to the reinforcer in the nondeprived state, however, provides the animals with an opportunity to learn about the relatively low value of the reinforcer when they are nondeprived, and thus they can assign an appropriate incentive value when once again they find themselves in this state.

Although in earlier studies researchers have used instrumental procedures to analyze the influence of different levels of water deprivation at the time of learning on extinction or relearning (see Hillman, Hunter, \& Kimble, 1953; Kendler, 1945), there have been no previous attempts to assess the effect of a posttraining shift in water deprivation in the absence of prior experience with the reinforcer under the test motivational state. Consequently, the aim of the present experiments was to investigate the role of incentive learning in the motivational control of instrumental performance following a posttraining shift from a high to a low level of water deprivation in an attempt to assess the generality of incentive learning in within-state motivational shifts.

\section{EXPERIMENTS 1A AND 1B}

In the first stage of the present experiments, all animals were exposed to an alternating schedule of high and low levels of water deprivation. During this stage, half of the animals were given noncontingent exposure to the instrumental reinforcer on days on which they were thirsty, and the remainder received presentations of the reinforcer while they were relatively sated. Following the preexposure sessions, all animals were trained to press the lever while in the high-deprivation state. After the final training session, half of the animals that were preexposed under thirst and half that were preexposed when they were sated were maintained in the high motivational state, while the remainder again sated themselves with water before instrumental performance was assessed in a single extinction test.

If the motivational control of performance is mediated by a process of incentive learning, the instrumental performance of animals trained in the high-deprivation state and shifted to the low state on test should persist at a level similar to that of animals tested in the high-deprivation state, unless the former were previously allowed to contact the reinforcer in the low-deprivation state. By contrast, if instrumental performance is a direct function of the degree of deprivation, as traditional theories of motivation assume (e.g., Hull, 1943), then extinction performance of both the high-deprivation groups should be greater than that of the groups tested in the low-deprivation state.

In Experiments $1 \mathrm{~A}$ and $1 \mathrm{~B}$, the procedural details were the same, except that the instrumental reinforcers used in each case differed so that we could examine the generality of any effects of preexposure. In Experiment 1A, the instrumental reinforcer was a lemon-flavored maltodextrin solution, whereas in Experiment 1B, it was a quinine-flavored sucrose solution.

\section{Method}

Subjects and Apparatus. There were 16 adult male hooded Lister rats in each experiment. They were housed in groups of 4 . Training and testing took place in four Campden Instruments operant chambers housed in sound and light-resistant shells. Each chamber was equipped with a dipper, which could deliver $0.05 \mathrm{ml}$ of a 20\% solution of "snowflake" maltodextrin (Cerestar Ltd, Manchester, England), a complex polysaccharide, flavored with $3 \%$ lemon juice, or a $20 \%$ sucrose solution flavored with $0.0125 \%$ quinine hydrochloride. Both reinforcers were delivered into a recessed magazine, which rats could enter through a flap door positioned in the center of the front wall. A retractable lever was located to the right of this flap door. Each chamber was illuminated by a 3-W, 24-V houselight mounted on the front wall above the magazine flap 
door. A BBC microcomputer equipped with the SPIDER extension (Paul Fray Ltd., Cambridge, England) controlled the equipment and recorded the leverpresses during instrumental training and testing.

The preexposure phase of these experiments was conducted in four plastic cages located in another room. These cages were black plastic boxes ( $30 \mathrm{~cm}$ long $\times 13 \mathrm{~cm}$ wide $\times 15 \mathrm{~cm}$ high) with wire mesh ceilings and were fitted with glass drinking tubes from which fluid was delivered via a metal drinking spout inserted into each box through a hole in the front wall.

Procedure. During the preexposure stage of both experiments, all of the animals were placed on an alternating schedule of high $(22.5 \mathrm{~h})$ and low (free access) levels of water deprivation for 6 days. Half of the animals in each experiment, Groups High/High and High/Low, were given preexposure to the solutions that would act as the instrumental reinforcer on Days 1, 3, and 5, while they were under the high level of water deprivation. Four hours after each of these sessions, the animals in these groups received free access to tap water in their home cages. The remaining subjects, Groups Low/High and Low/Low, received identical preexposure to the reinforcers while they were sated on Days 2, 4, and 6. Immediately before the start of these sessions, the water was withdrawn from the home cages and the preexposure session began. During the preexposure sessions, the subjects were placed in the preexposure cages fitted with glass drinking tubes and were removed 30 min later. For animals in Experiment $1 \mathrm{~A}$, the drinking tubes contained the lemon-flavored maltodextrin solution, whereas for the animals in Experiment 1B, the drinking tubes contained the quinine-flavored sucrose solution. Four hours after the final preexposure session, all animals were given 1.5-h free access to water and immediately placed on a 22.5-h water deprivation schedule, under which they received 1.5 -h access to water in the home cages after each training session. Throughout the preexposure stage and the rest of the experiment, food was freely available in the home cages.

The instrumental training sessions were the same in both experiments, except that in Experiment 1A, the reinforcer was the maltodextrin solution, whereas in Experiment 1B, it was the sucrosequinine solution. Initially, all of the animals received two $30-\mathrm{min}$ sessions of magazine training, in each of which the appropriate reinforcer was delivered on a random time 60 -sec schedule with the levers retracted. Following magazine training, all of the animals received three instrumental training sessions in which leverpressing was reinforced on a random interval schedule whose parameter was increased from 2 through 15 to $30 \mathrm{sec}$ across successive sessions. After one further session on the random interval $30-\mathrm{sec}$ schedule, training concluded. Each training session started with the insertion of the lever and ended with its retraction 30 min later. Immediately after the final training session, the subjects in Groups High/Low and Low/Low were returned to the free-water schedule. The subjects in the other groups, Groups High/High and Low/High, were maintained on the 22.5 -h water deprivation schedule. The effect of this posttraining shift in primary motivation was assessed in a 30-min extinction session on the next day, during which no reinforcers were presented. The test session started with the insertion of the levers and ended with their retraction after $30 \mathrm{~min}$.

Finally, to assess the effectiveness of the posttraining shift in deprivation on instrumental performance in the presence of the reinforcers, a final 30-min reacquisition session was conducted under the test deprivation state, in which leverpressing was again reinforced with the appropriate reinforcer on a random interval $30-\mathrm{sec}$ schedule.

\section{Results and Discussion}

The number of leverpresses in the extinction test was initially evaluated with a two-way analysis of variance, distinguishing between performance in the two experiments and in the four groups. Although the performance varied significantly between the groups $[F(3,24)=3.42$, $p<.05$ ], the $F$ ratios for the main effect of experiment and its interaction with group were both less than one. Consequently, the results of Experiments $1 \mathrm{~A}$ and $1 \mathrm{~B}$ were combined for presentation and analysis.

The left-hand panel of Figure 1 displays the results of primary interest, the number of leverpresses during the extinction test. The data essentially replicate the pattern observed by Balleine (in press) for a decrease in the level of food deprivation between training and testing. When our animals were tested under high water deprivation, performance was unaffected by the level of deprivation employed during preexposure to the reinforcer. More importantly, in the absence of preexposure to the reinforcer in the low-deprivation state, a shift to this state in testing did not reduce leverpressing in relation to the performance of the animals tested under the high-deprivation state; if anything, Group High/Low tended to press more than Groups Low/High and High/High. Finally, the fact that the animals in Group Low/Low, who were both tested and preexposed in the low-deprivation state, pressed less than any of the other groups replicates Balleine's finding that sensitivity to a motivational shift depends on prior exposure to the reinforcer in the test state.

The reliability of this pattern of test performance was evaluated in a series of preplanned orthogonal contrasts that were based on the predictions derived from Balleine's (in press) results. Neither the difference between Groups Low/High and High/High $(F<1)$ nor the difference between this pair of groups and Group High/Low $[F(1,24)=3.86, p>.05]$ was significant. By contrast, the difference between Group Low/Low and the remaining three groups was reliable $[F(1,24)=6.36, p<.02]$.

The interpretation of this significant contrast is not compromised by baseline differences between the groups in the rate of leverpressing on the last training session $(F<1)$. The mean number of leverpresses emitted on the last training session was, for Group Low/Low, 314; for Group High/Low, 271; for Group Low/High, 293; and for Group High/High, 301. In fact, the same pattern

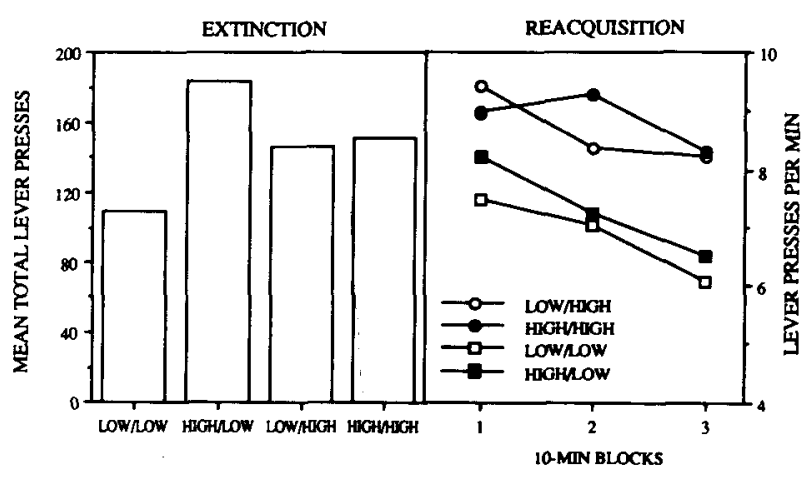

Figure 1. Experiments 1A \& 1B: Mean number of leverpresses during the extinction test session (kef-hand panel), and leverpresses per minute during the reacquisition test in 10-min blocks (right-hand panel), for each of the groups. The first term in each group's label refers to the deprivation state during the preexposure phase and the second one refers to the test deprivation state. 
of test performance is observed when the number of leverpresses in the extinction test is expressed as a percentage of the number in the last training session. The mean percentages are, for Group Low/Low, 40\%; for Group High/Low, $107 \%$; for Group Low/High, $66 \%$; and for Group High/High, 53\%. Again the contrasts between Group High/High and Low/High and between these groups and Group High/Low were not significant $(F s<1)$, whereas that between Group Low/Low and the remaining groups was reliable $[F(1,24)=4.84, p<.05]$.

Performance during the reacquisition test was analyzed to confirm that the shift to a low level of water deprivation did in fact reduce the reinforcing properties of the sucrose and maltodextrin solutions. The illustration of the mean rates of pressing during this reacquisition session in the right-hand panel of Figure 1 shows that on the average the animals in the low-deprivation state pressed less than those in the high state. Due to the large betweensubject variance, however, this difference was not significant $(F=1)$. But a reliable difference was observed when the number of presses in the reacquisition session was expressed as a percentage of those in the last training session $[F(1,24)=4.88, p<.05]$, an effect that did not interact with that of the preexposure state $[F(1,24)=$ $1.25, p>.20]$. These percentage scores were, for Group Low/Low, $71 \%$; for Group High/Low, $77 \%$; for Group Low/High, 96\%; and for Group High/High, 85\%.

In summary, these experiments replicate for a reduction in level of water deprivation the pattern of results observed by Balleine (in press) for a comparable transition between levels of food deprivation. In accord with incentive theory, a reduction in the level of water deprivation reduced instrumental performance in an extinction test only if the animals previously had the opportunity to learn about the value of the reinforcer in that state by being exposed to it when they were nondeprived.

\section{EXPERIMENT 2}

Expectancy theory argues that the change in the value of a reinforcer brought about by incentive learning is mediated by the agent's knowledge of the relationship between the instrumental action and the reinforcer. Although the results of the first two studies accord with this claim, the training procedure used in these experiments not only exposed the animals to the instrumental contingency between leverpressing and the reinforcer but also to a Pavlovian relationship between the contextual cues and the reinforcer. Thus, the effect of incentive learning could well have been mediated by the Pavlovian-reinforcer association rather than the instrumental contingency. The role of the instrumental contingency in mediating the incentive learning effect was examined in the present study by using a concurrent training procedure.

Again, as in Experiments $1 \mathrm{~A}$ and 1B, all animals were initially exposed to an alternating schedule of low and high levels of water deprivation. During this stage, half of the animals were preexposed to the lemon-flavored maltodex- trin solution on days when they were in the high water deprivation state and to the quinine-flavored sucrose solution on days when they were in the low-deprivation state, with the remaining animals experiencing the opposite assignment. Following this preexposure period, all animals were maintained on a 22.5 - $\mathrm{h}$ water deprivation schedule and trained to press a lever and pull a chain, with delivery of the sucrose solution contingent on one action and the maltodextrin solution contingent on the other. After the final training session, all of the animals were given unlimited access to water in their home cages and their performance on the lever and chain was assessed in an extinction test in the absence of the reinforcers. The instrumental expectancy account predicts that the preexposure phase, by influencing the incentive values assigned to the reinforcers, will produce a relative reduction in performance of the action trained with the reinforcer preexposed in the low-deprivation state when the extinction test is conducted in that state. By contrast, accounts which argue that the associations between the contextual cues and the reinforcer can modulate instrumental performance would anticipate that, since these stimuli were equally associated with both reinforcers during training, the performance of the two actions should not differ in extinction following a shift to the low-deprivation state.

\section{Method}

Subjects and Apparatus. The subjects were 36 male hooded Lister rats housed under the same conditions as in the previous experiments and trained in the same chambers. In this study, however, each chamber was also equipped with a chain, which could be lowered through the roof from a microswitch so that it hung to the left of the magazine flap door. Thus, the lever and the chain were positioned symmetrically to the right- and left-hand sides of the magazine flap door, respectively. Each chamber was also equipped with a dipper that could deliver $0.05 \mathrm{ml}$ of the $20 \%$ solution of sucrose flavored with $0.0125 \%$ of quinine hydrochloride and a peristaltic pump that could deliver $0.05 \mathrm{ml}$ of the $20 \%$ solution of maltodextrin, flavored with $3 \%$ lemon juice. The plastic cages used during the preexposure phase were the same as those employed in Experiments $1 \mathrm{~A}$ and $1 \mathrm{~B}$.

Procedure. As in the previous studies, all of the animals were initially placed on a schedule of either a high or a low level of water deprivation, with deprivation states alternating daily. During the preexposure phase, which lasted 6 days, the animals were placed in the preexposure cages containing the glass drinking tubes and were given access to the appropriate solution for $30 \mathrm{~min}$. On Days 1,3 , and 5, while in the high-deprivation state, half of the animals received the quinine-flavored sucrose solution, whereas the remaining animals were preexposed to the lemon-flavored maltodextrin solution. Four hours after the completion of these sessions, all of the animals were given free access to water in their home cages. By contrast, on Days 2, 4, and 6, while in the low-deprivation state, all of the animals received exposure to the alternative solution to the one they were exposed to while thirsty. Following the final preexposure session, all of the animals were placed on a 22.5-h water deprivation schedule with $1.5 \mathrm{~h}$ of free access to water in the home cages. This deprivation schedule was maintained throughout the instrumental training session.

The training sessions began the next day. Initially, all subjects received three sessions of magazine training, in each of which 15 presentations of the sucrose solution and 15 presentations of the maltodextrin solution were delivered in a random order on a ran- 
dom time 60-sec schedule, with the levers and chains withdrawn. Following the magazine training sessions, all of the animals were trained to chain pull in the absence of the lever during the first three sessions and then to leverpress in the absence of the chain in the next session. During these sessions, instrumental actions were reinforced on a random interval 2-sec schedule. Each instrumental session started with the onset of the houselight and ended with its offset $30 \mathrm{~min}$ later. Twelve rats failed to acquire chain pulling and were discarded from the experiment. For 13 of the remaining 24 rats, pressing the lever was reinforced with maltodextrin and pulling the chain was reinforced with the sucrose, with the other subjects receiving the opposite action-reinforcer assignment. Of the 13 rats assigned to the former action-reinforcer arrangement, 6 had been preexposed to the maltodextrin solution in the high-deprivation state and to sucrose solution in the low-deprivation state, whereas 7 rats had received the opposite preexposure assignments. Of the 11 rats assigned to the opposite action-reinforcer arrangement, 5 had been preexposed to the maltodextrin solution in the highdeprivation state and to sucrose in the low-deprivation state, whereas the 6 remaining animals had received the opposite preexposure treatments.

During concurrent training with both the lever and the chain, the sucrose and the maltodextrin solutions became available on a single random interval schedule. When a reinforcer became available, it was assigned to one of the two actions with equal probability. The subroutine controlling the schedule was suspended whenever a reinforcer became available and only restarted once it had been delivered. During each session, a total of 30 reinforcers were delivered, 15 of them contingent on one action and 15 on the other. As soon as the animals had obtained 15 of one type of reinforcer, this type was no longer delivered, and the schedule immediately programmed the interval to the next reinforcer. The session continued until 15 of the other reinforcers had been delivered, at which point it ended. This type of concurrent schedule ensures that on the average the reinforcers are evenly distributed to the two actions across a session, whatever the relative distribution of performance between the two actions. The nominal schedule parameters were 2 and $7 \mathrm{sec}$ for the first two sessions of concurrent training and $15 \mathrm{sec}$ for the next four sessions.

Immediately following the final training session, all of the animals were given free access to water, and on the next day, performance on the lever and chain was assessed in a single 30-min extinction session in the low-deprivation state and in the absence of any reinforcers.

\section{Results and Discussion}

The total number of leverpresses and chain pulls during training was evaluated by a three-way mixed analysis of variance. Two between-subject factors, reinforcer and manipulandum, distinguished between the two reinforcers preexposed in the low-deprivation state and between the two actions reinforced by that reinforcer during training, respectively. The within-subject preexposure factor distinguished between performance on the manipulandum reinforced by the incentive preexposed in the lowdeprivation state from that on the other manipulandum. An analysis of leverpress and chain-pull performances during the final concurrent training session revealed no significant effect of preexposure $(F<1)$, nor any significant interaction between this factor and the effects of reinforcer and manipulandum $[F(1,20)<3.90 ; p>.05$ in all cases]. In the final training session, animals performed the action reinforced with the incentive preexposed in the low-deprivation state at a rate of 6.0 responses per minute as opposed to a mean of 6.2 responses per minute

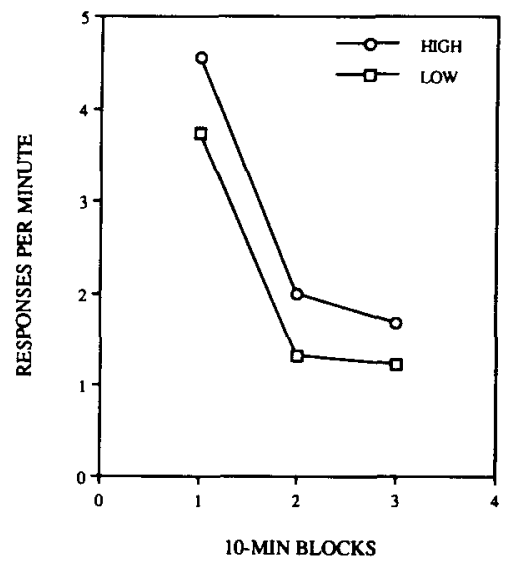

Figure 2. Experiment 2: Mean number of responses per minute on the leverpress and chain-pull manipulanda during the extinction test. Separately presented are performance of the action reinforced with the incentive preexposed in the high-deprivation state $(H I G H)$ and performance of the action reinforced with the incentive preexposed in the low-deprivation state (LOW).

for the action reinforced with the incentive preexposed in the high-deprivation state.

The results of central importance in this experiment are those relating to the extinction test. Figure 2 displays the mean number of responses per minute on the manipulandum reinforced with the incentive preexposed in the lowdeprivation state and the number of responses on the manipulandum reinforced with the incentive preexposed in the high-deprivation state, for successive 10-min blocks. This figure indicates that performance of the action trained with the incentive preexposed in the low-deprivation state was consistently at a lower rate than performance of the action reinforced with the incentive preexposed in the high-deprivation state.

The preceding description of the data was confirmed by the statistical analysis conducted on performance during the extinction test. This analysis revealed a significant effect of the preexposure factor $[F(1,20)=5.18$, $p<.05]$, but no effect of the reinforcer $[F(1,20)=1.92$, $p>.10]$ or manipulandum factors $[F(1,20)=3.01$, $p>.05]$. There were no significant interactions between the preexposure factor and the reinforcer $[F(1,20)=$ $1.45, p>.10]$ or the manipulandum factors $[F(1,20)=$ $3.06, p>.05]$.

In conclusion, the pattern of results observed in this experiment confirms the predictions of incentive theory. The effect on instrumental performance of a revaluation of the reinforcer by a posttraining shift in primary motivation from a high to a low level of water deprivation was found to depend on previous exposure to the reinforcer in the low-deprivation state. Moreover, the present study established that this effect of preexposure was mediated by the instrumental contingency in force during training. Preexposure to a reinforcer in the low-deprivation state produced a selective reduction in the performance of the action upon which delivery of that reinforcer was contingent during training. Thus, in accordance with expectancy the- 
ory, motivational control of an instrumental action following a posttraining shift in primary motivation depends on the animal's knowledge about the action-outcome relationship and the incentive value assigned to the outcome on the basis of previous experience with the reinforcer in the test motivational state.

\section{GENERAL DISCUSSION}

The pattern of results observed in the present experiments supports the conclusion that control of instrumental performance following a posttraining shift in primary motivation is determined both by the animal's knowledge about the action-reinforcer relationship and by the current incentive value that the animal assigns to the reinforcer, a value that is determined by a process of incentive learning. Experiments $1 \mathrm{~A}$ and $1 \mathrm{~B}$ demonstrated that, in the absence of an incentive learning treatment, instrumental performance was not affected by a posttraining shift from a high to a low level of water deprivation. If, however, animals previously had the opportunity to consume the reinforcer in the test deprivation state, the low-deprivation level, then their performance was altered appropriately when they were shifted to that state. Experiment 2 , in which a concurrent training procedure was used, demonstrated that sensitivity of instrumental performance to a posttraining shift in primary motivation depends on the animal's knowledge of the outcome that was contingent on the action during training. The preexposure experience with the incentives produced a relative reduction in performance of the action reinforced with the incentive preexposed in the low-deprivation state when extinction was conducted in that state.

The present results add to the growing body of evidence that the motivational control of instrumental actions is mediated by incentive learning. As well as the studies of Balleine (in press) and Dickinson and Dawson (1988, 1989), who used shifts between different levels of hunger and between hunger and thirst, Hendersen and Graham (1979) reported a comparable effect in the case of heat avoidance. Having trained rats to avoid a heat source in a hot environment, they then tested performance in extinction when the ambient temperature was low. Although the reduction in ambient temperature would be expected to shift the incentive value of the heat lamp from negative to positive, the performance of the avoidance response was in fact unaffected by the ambient temperature during test. To reduce the level of avoidance responding in the cold environment, preexposure to noncontingent presentations of the heat lamp under the low ambient temperature was required. Finally, the role of incentive learning is not restricted to the control of incentive value by motivational states. Balleine and Dickinson (1991) have recently reported that the devaluation of a reinforcer by flavor aversion conditioning only affected subsequent instrumental performance in extinction if animals had received prior reexposure to the devalued reinforcer.
Although the importance of incentive learning in the motivational control of instrumental performance is well established, what remains less clear is the nature of the incentive learning process itself. Revusky (1968) has reported that rats consume relatively more of a flavored solution to which they have been exposed under a high rather than a low level of water deprivation. In contrast to the present incentive learning effect, however, this preference was independent of the deprivation state at the time of testing. In our studies, as in those by Balleine (in press), preexposure under the low-deprivation state had no detectable effect on performance when testing occurred under the high state.

Cabanac (1971) has suggested that the hedonic reactions to a commodity are modulated by the agent's current motivational state, and thus, if the incentive value of a reinforcer is determined by the hedonic reaction that it elicits, this modulation would provide a process by which the agent could assign incentive values that were conditional upon motivational states. Although we know of no direct evidence for the modulation of hedonic reactions by thirst, there is evidence for such a process for other motivational states. Berridge (1991) has recently reported that the ingestive reactions elicited in rats by nutritive solutions are enhanced by food deprivation. Because such ingestive reactions are usually interpreted as reflecting the hedonic properties of the stimulus (Grill \& Berridge, 1985), it is tempting to conclude that these properties are in part determined by the agent's current motivational state. A comparable modulation of the ingestive reactions to saline by varying states of sodium depletion has also been reported (Berridge, Flynn, Schulkin, \& Grill, 1984).

Whatever the merits of this hedonic theory of incentive value, it is clear from the present and related studies that primary motivational states, such as thirst, exercise minimal direct control over goal-directed, instrumental actions; rather, such control is mediated by a process of incentive learning whereby the agent assigns incentive values that are conditional upon motivational states.

\section{REFERENCES}

BALleine, B. (in press). Instrumental performance following a shift in primary motivation depends upon incentive learning. Joumal of Experimental Psychology: Animal Behavior Processes.

Balleine, B., \& Dickinson, A. (1991). Instrumental performance following reinforcer devaluation depends upon incentive leaming. Quarterly Journal of Experimental Psychology, 43B, 279-296.

Berridge, K. C. (1991). Modulation of taste affect by hunger, caloric satiety, and sensory-specific satiety in the rat. Appetite, 16, 103-120.

Berridge, K. C., FlynN, F. W., Schulkin, J., \& Grill, H. J. (1984). Sodium depletion enhances salt palatability in rats. Behavioral Neuroscience, 98, 652-660.

Cabanac, M. (1971). Physiological role of pleasure. Science, 173, 1103-1107.

Dickinson, A. (1989). Expectancy theory in animal conditioning. In S. B. Klein \& R. R. Mowrer (Eds.), Contemporary learning theories: Pavlovian conditioning and the status of traditional learning theory (pp. 279-308). Hillsdale, NJ: Erlbaum. 
Dickinson, A., \& Dawson, G. R. (1988). Motivational control of in strumental performance: The role of prior experience of the reinforcer. Quarterly Journal of Experimental Psychology, 40B, 113-134.

Dickinson, A., \& DAwson, G. R. (1989). Incentive learning and the motivational control of instrumental performance. Quarterly Journal of Experimental Psychology, 41B, 99-112.

GrILL, H. J., \& BERRIDGE, K. C. (1985). Taste reactivity as a measure of the neural control of palatability. Progress in Psychobiology \& Phys iological Psychology, 11, 1-61.

Hendersen, R. W., \& Graham, J. (1979). Avoidance of heat by rats: Effects of thermal context on rapidity of extinction. Learning \& Motivation, 10, 351-363.

Hillman, B., Hunter, W. S. Kimble, G. A. (1953). The effect of drive level on the maze performance of the white rat. Journal of Comparative \& Physiological Psychology, 46, 87-89.
Hull, C. L. (1943). Principles of behavior. New York: AppletonCentury-Crofts.

KENDLER, H. H. (1945). Drive interaction: II. Experimental analysis of the role of drive in learning theory. Journal of Experimental Psychology, 35, 188-198.

REVUSKY, S. H. (1968). Effects of thirst level during consumption of flavored water on subsequent preference. Journal of Comparative \& Physiological Psychology, 66, 777-779.

Tolman, E. C. (1949a). The nature and functioning of wants. Psychological Review, 56, 357-369.

Tolman, E. C. (1949b). There is more than one kind of learning. Psychological Review, 56, 144-155.

(Manuscript received December 18, 1991; revision accepted for publication March 28, 1992.) 